\title{
Design, test, and calibration of an electrostatic beam position monitor
}

\author{
Maurice Cohen-Solal \\ Laboratory of the Linear Accelerator (LAL), CNRS/University, F-91406 Orsay, France
}

(Received 1 October 2008; published 16 March 2010)

\begin{abstract}
The low beta of proton or ion beams favors an electrostatic pickup to measure the transverse beam centroid position. Often papers on beam position monitors (BPM) are focused on a particular aspect of the problem; however, it is important to consider all various issues of a position measurement system. Based on our experience at the IPHI (high intensity injector proton) facility at CEA-Saclay, this paper will address all aspects to design, test, and calibrate a BPM for proton linear accelerators, while emphasizing the determination of the absolute beam position. We present details of the readout electronics, and describe the calibration of the BPM using a test station. For calculation and simulation of the electrical signals we developed a MATHEMATICA script. The error analysis presented, on the basis of six BPMs installed in the high energy section of IPHI, demonstrates the expected accuracy of the position measurement. These studies also identify the parameters that could improve the performance of the beam position control. The experience from these developments is currently being used for the BPM design and test stand dedicated to the Spiral2 accelerator at Ganil-Caen which will deliver heavy ion beams.
\end{abstract}

DOI: 10.1103/PhysRevSTAB.13.032801

PACS numbers: 29.90.+r

\section{INTRODUCTION}

For a low beta velocity beam $(\beta<0.1)$, the capacitive beam position monitor (BPM) pickup is more efficient in terms of output signal power, than an inductive one of the same physical size [1]. The presented BPM uses four capacitive-coupling electrodes, called "buttons." The high energy beam line of the high intensity injector (IPHI) [2] is equipped with BPMs of two different sizes: five fit into the vacuum chamber of $33 \mathrm{~mm}$ radius, and one is located in the large aperture chamber of $75 \mathrm{~mm}$ radius, just in front of the beam stopper. The IPHI aims to produce a continuous wave $(\mathrm{CW})$ high intensity proton beam of $100 \mathrm{~mA}$ at $3 \mathrm{MeV}$, at a bunching frequency of $352.2 \mathrm{MHz}$. The design and implementation of the BPM diagnostic needs many considerations: signal processing and computation, mechanics, signal transmission and cables, electronics, noise issues, sensitivity, calibration, and numerous tests. This paper discusses all of these points in a single report. While for storage rings and synchrotron light sources a high resolution and control of the beam orbit is achieved-typically $1 \mu \mathrm{m}$, or better-a linear accelerator needs a high accuracy of the absolute values reported by the transverse beam position measurement system. One of the major reasons regarding the design issues lies in the fact of a poor transfer sensitivity of the BPM pickup itself. A large BPM chamber radius has intrinsically a low sensitivity. The IPHI BPM sensitivity is $2 \% / 100 \mu \mathrm{m}$, which compares to a required $\pm 100 \mu \mathrm{m}$ total accuracy. Based on the recent experience, this paper discusses all the considerations required to deliver accurate results from the beam position measurement of low beta, high intensity beams.

\section{ELECTRODE SIGNAL}

\section{A. Theory}

The current signal of the pickup electrode can be expressed versus the line charge density $\lambda(z, t)$ or the beam current $i_{b}(t)$ :

$$
\begin{aligned}
& i_{\text {button }}(t)=\frac{d}{d z} \lambda(z, t) \beta c \frac{\text { area }_{\text {button }}}{\text { circum }_{\text {pipe }}} \\
& i_{\text {button }}(t)=\frac{d}{d t} i_{b}(t) \frac{1}{\beta c} \frac{\text { area }_{\text {button }}}{\text { circum }_{\text {pipe }}} .
\end{aligned}
$$

These expressions can be derived from the electrostatic field $[1,3]$ or more classically from the image charge of the beam. Often a $\cos ^{2}$ or a Gaussian longitudinal particle distribution is used. These two expressions are equivalent, and are obtained by applying the electrostatic case: bunch length (seen by the electrode) $\gg$ electrode length. The first equation expresses the electrode current signal in terms of the charge density $\lambda$ and beam velocity $\beta c$. The second form is used more, as it applies the beam current as a control parameter.

The frequency domain voltage signal of the button electrode $v_{\text {button }}(\omega)$ is expressed by the product of the button current signal $i_{\text {button }}(\omega)$ and the load impedance seen by the electrode, i.e., the parallel network of $Z_{c}=50 \Omega$ transmission line (cable impedance) and the electrode capacitance $C_{b}$ [4]:

$$
v_{\text {button }}(\omega)=\frac{Z_{c}}{1+Z_{c} C_{b} j \omega} i_{\text {button }}(\omega),
$$

$Z_{c}$ is the cable impedance. The convolution of this product 


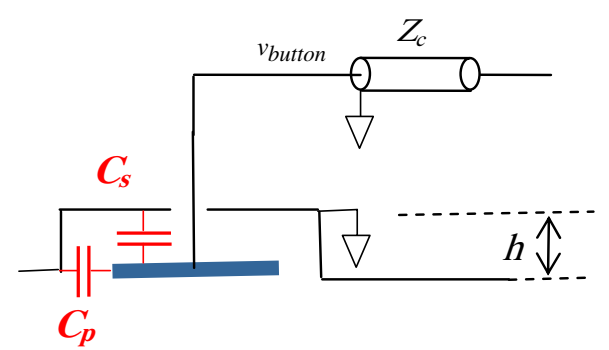

FIG. 1. (Color) Electrical button model, $C_{S}$ is the capacitance formed by the surface of the electrode and the grounded wall, and $C_{p}$ is the button radial capacitance. $C_{b}=C_{s}+C_{p}$ is the total electrode capacitance. $h$ is the distance between the electrode surface and the wall.

gives the button voltage signal in the time domain, referring to [3].

The button pickup has a high-pass-like characteristic, with different behavior of the two regions separated by the cutoff frequency $f_{c}=1 /\left(2 \pi Z_{c} C_{b}\right)$ (Fig. 2). At low frequencies $f \ll f_{c}, v_{\text {button }}$ is independent of the button capacitance $C_{b}$ (and therefore of the distance $h$ ), but depends only on the electrode surface area $a_{\text {button }}$ [Eqs. (1a) and (1b)]. It is important to notice the dependence to the load impedance $Z_{c}$, which results in a change of the transfer characteristics if the cable/electronics input impedances vary. The time structure of the button voltage signal appears as a derivative of the longitudinal bunch shape (particle distribution), seen at the surface of the vacuum chamber wall if the electrostatic condition applies: bunch rms length $\gg$ button electrode length [Eq. (1)].

At high frequencies $f \gg f_{c}$, with Eq. (1) inserted into Eq. (2), the button voltage signal is independent of the load impedance $Z_{c}$ :

$$
v_{\text {button }}(\omega)=\frac{1}{C_{b} \omega} \omega i_{\text {button }}(\omega) \frac{\text { area }_{\text {button }}}{\text { circum }_{\text {pipe }}} .
$$

In this case, the time structure of the button voltage signal is identical to the longitudinal particle distribution

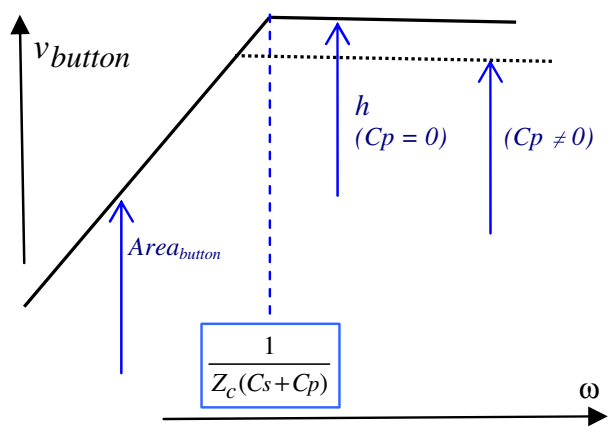

FIG. 2. (Color) Asymptotes of the button transfer function. The gain is proportional to the distance $h$ at high frequencies and to the electrode surface at low frequencies. The cutoff frequency is a function of the total capacitance. A modification of the area and/or $h$ changes also the capacitance and therefore the cutoff frequency. of the bunch seen at the vacuum chamber wall. For $C_{p}=0$ and $C_{b}=\varepsilon_{0} \frac{\text { areautton }}{h}$ in Eq. (3), we observe that $v_{\text {button }}$ is independent of the button capacitance $C_{b}$, but is only proportional to the distance $h[3,4]$. Figure 2 summarizes these results and Fig. 3 shows the measured frequency domain characteristic of the button electrode.

Transfer function measurements of a button BPM in a coaxial-line test arrangement are in good agreement with the theory [Fig. 3(a)]. The sensitivity of the IPHI BPM is $10.7 \mathrm{mV} / \mathrm{mA}$ beam current at $352 \mathrm{MHz}$. The two regions [Fig. 3(a)] are separated by the low cutoff frequency $707 \mathrm{MHz}$ corresponding to the time constant $4.5 \mathrm{pF} \times$ $50 \Omega$.

A modification of $C_{s}$ has a direct effect on $f_{c}$ and an indirect effect on the gain via the variations of area ${ }_{\text {button }}$ and/or $h . C_{p}$ is a parasitic capacitance that reduces the cutoff frequency and the gain at high frequencies (Fig. 2).

High frequency information from the BPM is sometimes required to estimate bunch length or shape characteristics, or for the monitoring of the beam phase/time-of-flight. The transfer response of the button electrode in Fig. 3(a) does not clearly indicate a high frequency roll-off; however, there are irregularities and resonant effects at very high frequencies due to mismatching between beam pipe and BPM block, as well as beam pipe waveguide resonances. Moreover, mismatches between the characteristic impedance of the cables and electronics inputs also affect the response at high frequencies. On the BPM side, the cable is terminated by the feedthrough-button electrode transmission line system. The electrode impedance should be purely capacitive. The mechanical connection between the $50 \Omega$ feedthrough and the electrode is equivalent to a serial inductance, which can introduce some ringing of the signal. The feedthrough and its termination can be ana-

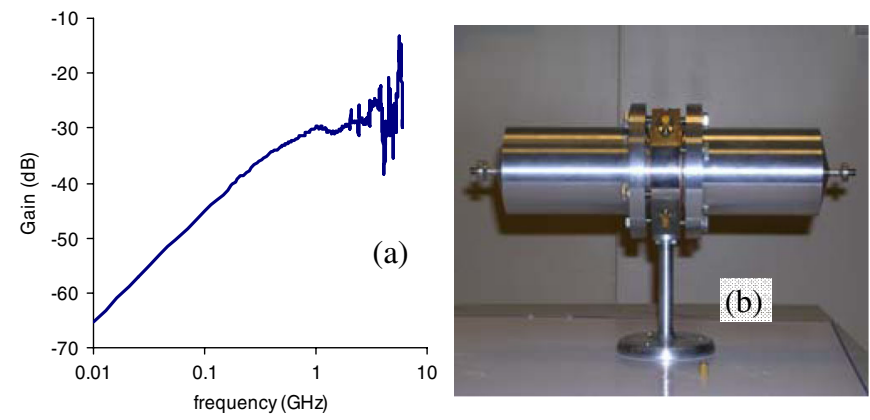

FIG. 3. (Color) (a) The measured transfer function of an IPHI BPM button. The gain is the ratio of the button voltage to the voltage injected into the $50 \Omega$ adapted pipe. (b) The cutoff frequency $707 \mathrm{MHz}$ corresponds to the time constant $4.5 \mathrm{pF} \times$ $50 \Omega$. The ripples around $4 \mathrm{GHz}$ are due to the bad mechanical adaptation between the tube and the BPM block. The characteristic impedance along the line is not suitable for high frequencies. Nevertheless, we do not see a frequency limitation up to $6 \mathrm{GHz}$ and never have reached the high cutoff frequency of the BPM itself. 
lyzed with a time-domain reflectometer (TDR) [5]. The TDR sends a step pulse down the feedthrough and returns a time-domain S11 impedance measurement. The terminal capacitance is deducted from the rise time, the discontinuities spot the physical location of impedance mismatches, and the eventual ringing indicates parasitic inductance [6].

Instead of the typical $50 \Omega$ electronics load impedance, a high impedance termination of the button electrode results in a higher gain at low frequencies [7]. High impedance electronics of this kind may be used for capacitive probe signal processing, for BPM signal processing it adds a substantial noise floor and cannot used at high frequencies. Moreover, it is difficult to match four electronics channels in gain and high impedance. A mismatch of only $2 \%$ corresponds to a $100 \mu \mathrm{m}$ position error, which cannot be tolerated because of the requirements.

\section{B. Simulation}

An extended MATHEMATICA model has been assembled and can be used when the electrostatic case is not always respected. This is often in the case of ions and protons accelerators where the bunch length is not much longer than the longitudinal length $L$ of the electrode, e.g., at the IPHI radio frequency quadrupole (RFQ) output, the bunch longitudinal extension is $24 \mathrm{~mm}$ for $L=22 \mathrm{~mm}$.

In the model developed, the longitudinal bunch shape is a $\cos ^{2}$ or a Gauss particle distribution. The current signal of the button is found by integrating the mirror charges on the electrode length $L$, Eq. (1) is replace by Eq. (4),

$$
i_{\text {button }}(t)=\int_{(-L / 2) / \beta c}^{(+L / 2) / \beta c} \frac{d}{d t} i_{b}(t) d t \frac{\operatorname{arc}_{\text {button }}}{\text { circum }_{\text {pipe }}},
$$

where $\operatorname{arc}_{\text {button }}$ is the radial aperture of the electrode expressed in units of length. Equation (4) allows an estimation of the signal characteristics for any combination of button and bunch length, and bunch shape (Fig. 4).

In the case of electrode length $L<$ bunch length, only a fraction of the image charge is integrated in the electrode, and the peak-to-peak electrode signal decreases when further reducing the electrode length $L$. The device operates below the cutoff frequency $f_{c}$; the signal is then a perfect time derivation of the bunch image charge distribution [Fig. 4(b)]. On the other hand, at electrode length
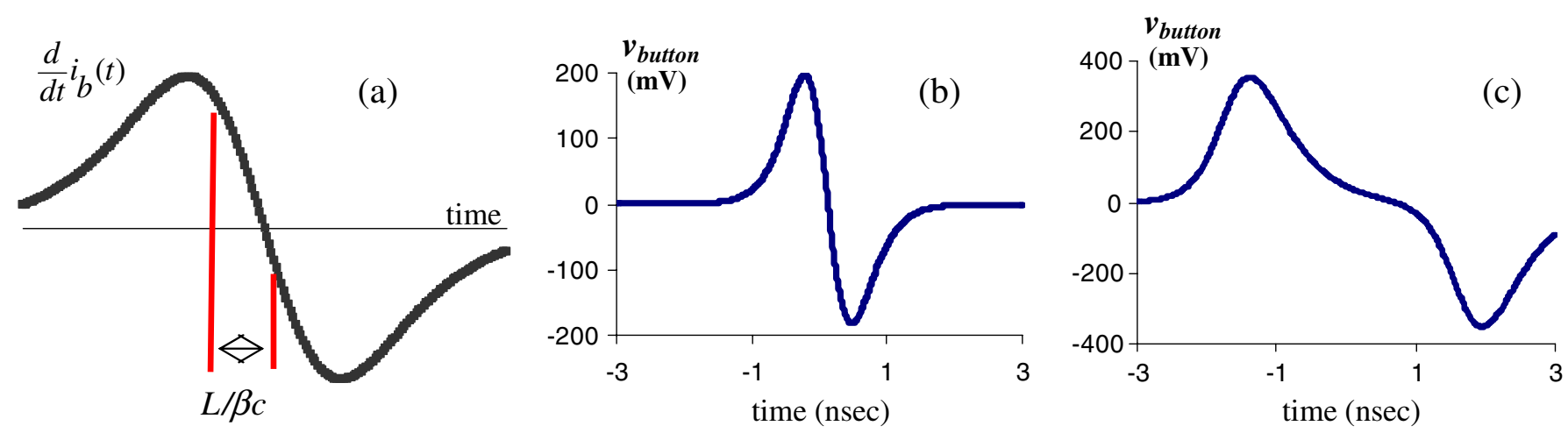

FIG. 4. (Color) (a) Simulation principle of the button current $i_{\text {button }}(t)$ with respect to Eq. (4). (b) and (c) The response function, computed with a MATHEMATICA routine, of two different button lengths, $L=10$ and $120 \mathrm{~mm}$ for a typical bunch of $\sigma_{\text {seen }}=17 \mathrm{~mm}$ of the superconducting linac of Spiral2. The (b) response (electrostatic case) appears as derivative of the bunch seen. The (c) response is identical to the longitudinal bunch shape. The pulse is positive when the bunch is entering inside the electrode and negative when the bunch is leaving the electrode. The simulation allows the optimization of the electrode dimensions for any mode.

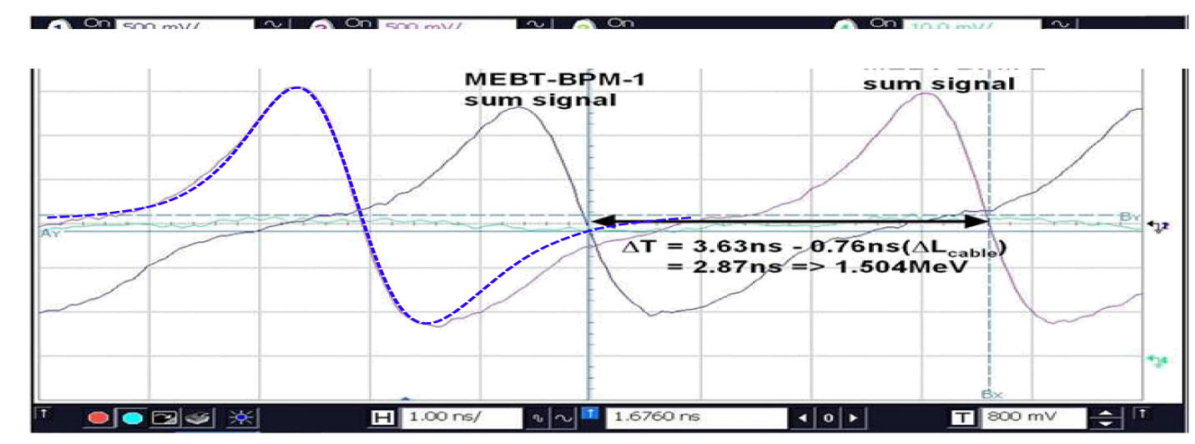

FIG. 5. (Color) Averaged sum of the four electrode signals obtained in the high energy section of SARAF. The dashed trace shows the response of a MATHEMATICA simulation using the same parameters as experimental conditions: a proton beam current of $4 \mathrm{~mA}$ at $\beta=0.06$, the bunch base length is $\pm 22^{\circ}$, the acceleration frequency is $176 \mathrm{MHz}$, and the inner pipe radius is $15 \mathrm{~mm}$. 


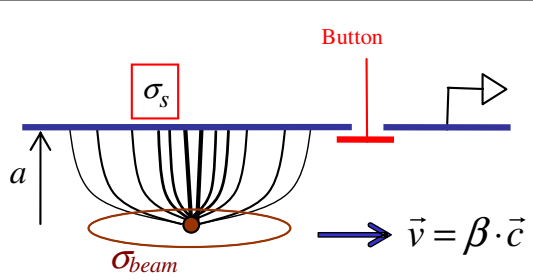

FIG. 6. (Color) A single charged particle moving at $\beta c$ creates an electrical field at the chamber wall of a longitudinal distribution $\sigma_{S}=\frac{a}{\gamma \sqrt{2}}$, where $\gamma$ is the relativist factor and $a$ is the radius chamber [9].

$L>$ bunch length the temporal signal length increases [Fig. 4(c)], and even may mix signals of consecutive bunches. Concerning the Spiral2 project, the electrode length is dimensioned to have a separation between signals coming from consecutive bunches.

Figure 5 compares the electrode signals obtained at the Soreq Applied Research Accelerator Facility (SARAF) installed in Israel and the corresponding MATHEMATICA computation.

\section{Image charge}

The electromagnetic wave associated with a low $\beta$ beam does not propagate in a pure transverse mode; the electromagnetic field has longitudinal components [8]. Therefore, the image charge distribution (opposite sign) [8] is wider than the physical length of the bunch charge distribution (Fig. 6).

The length of the image charge of the bunch is given by the quadratic sum $\sigma_{\text {im }}=\sqrt{\sigma_{s}^{2}+\sigma_{\text {beam }}^{2}}$ (Fig. 6), where $\sigma_{s}$ is the rms length of the image charge on the wall induced by a single particle and $\sigma_{\text {beam }}$ is the rms bunch length of the beam. This formula was experimentally tested on a capacitive probe at Ganil-Caen. Table I resumes the results obtained with a ${ }^{13} \mathrm{C}\left(Q / A=3^{+} / 6\right)$ beam of $1.5 \mu \mathrm{A}$ to $2.3 \mu \mathrm{A}$ at $12 \mathrm{MHz}$. The lengths of the image charge calculated are close to experimental observations except in the last case where the electronics bandwidth is limited.

TABLE I. The measured values are the temporal length of a base bunch seen by the capacitive probe. The base length is divided by 5.5 to obtain the rms value. The measurement was done by the Lecroy 7300A scope connected to the high impedance preamplifier output. It is known to have a limited bandwidth; therefore a measurement of bunch length $<5 \mathrm{nsec}$ (equivalent to $0.9 \mathrm{nsec}$ ) cannot be performed.

\begin{tabular}{lcccc}
\hline \hline$\beta$ & $\begin{array}{c}\lambda \\
\text { in } \mathrm{mm}\end{array}$ & $\begin{array}{c}\sigma_{\text {beam }} \\
\text { in mm }\end{array}$ & $\begin{array}{c}\sigma_{\text {im }} \\
\text { calculated }\end{array}$ & $\begin{array}{c}\sigma_{\text {im }} \\
\text { measured }\end{array}$ \\
\hline 0.041 & 1.0 & 22 & $32 \mathrm{~mm}, 2.7 \mathrm{nsec}$ & $2.7 \mathrm{nsec}$ \\
0.15 & 3.8 & 26 & $34 \mathrm{~mm}, 0.8 \mathrm{nsec}$ & $0.9 \mathrm{nsec}$ \\
0.38 & 9.4 & 43.5 & $48 \mathrm{~mm}, 0.5 \mathrm{nsec}$ & $0.9 \mathrm{nsec}$ \\
\hline \hline
\end{tabular}

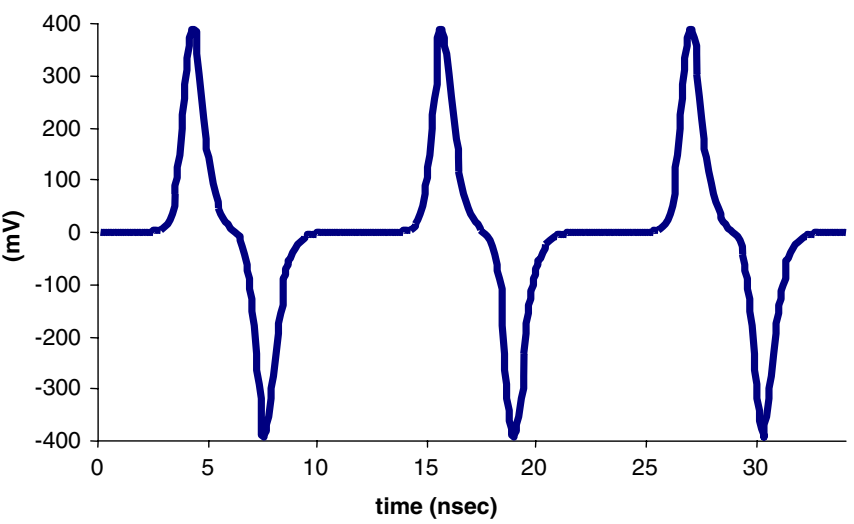

FIG. 7. (Color) The simulated temporal signal from the pickup designed for Spiral 2 linac. The aperture is $60^{\circ}$ and $L=39 \mathrm{~mm}$. This periodical signal can be decomposed in Fourier series. The first $(88.04 \mathrm{MHz})$ to the fifth coefficients are 127, 133, 41, 45, and $63 \mathrm{mV}$.

With the MATHEMATICA model, it is possible to expand the electrode voltage signal in its Fourier trigonometric series and, moreover, to pick a set of frequency harmonics for the signal evaluation (Fig. 7). The electrode length $L$ could be chosen to optimize the signal amplitude at the processing frequency.

In the case of IPHI, $\gamma=1.0032, \sigma_{s}=23 \mathrm{~mm}$, and $\lambda_{\text {beam }}=68 \mathrm{~mm}$, the induced charges in the wall have only little $\mathrm{AC}$ components, thus the resultant wall current is almost a coasting DC current (Fig. 8).

At $\gamma=1.003$ there is no relativistic effect, and the electric field in the electrode is like an electrostatic field $(\gamma=1)$. In the case of IPHI, the 2 nd and upper harmonics of the wall current are quasinull. Consequently, the electronics front end must operate at $352 \mathrm{MHz}$ with the interference risk from the rf power. Table II shows the effect of the particle distribution of the bunch- $\cos ^{2}$ or Gaussianfor the IPHI beam at the location of the first four BPM pickups. It will result in different levels of the first harmonic of the wall current, up to a factor 2 (second line in Table II). This is not a real problem because the electronics that we use has a large dynamic range, typically $70 \mathrm{~dB}$ or more.

When the FWHM of the bunch distribution is much higher than the period $\lambda_{\text {beam }}$, a pure mathematical calculation shows that the levels of harmonics become very dependent on the distribution. This is the case at the end of the high energy beam transport line, where the chamber radius is $75 \mathrm{~mm}, \sigma_{\mathrm{im}}=55 \mathrm{~mm}$ for $\lambda_{\text {beam }}=68 \mathrm{~mm}$ (fourth line in Table II). IPHI is a demonstrator project, the calculation of the dynamic range due to the longitudinal beam profile is not well understood for a high intensity proton beam. However, an uncertainty of the absolute beam position of $\pm 2 \mathrm{~mm}$ of BPM \#4 is tolerable to deliver the beam to the beam stopper. The real amplitude of the signals will be measured during the commissioning. 

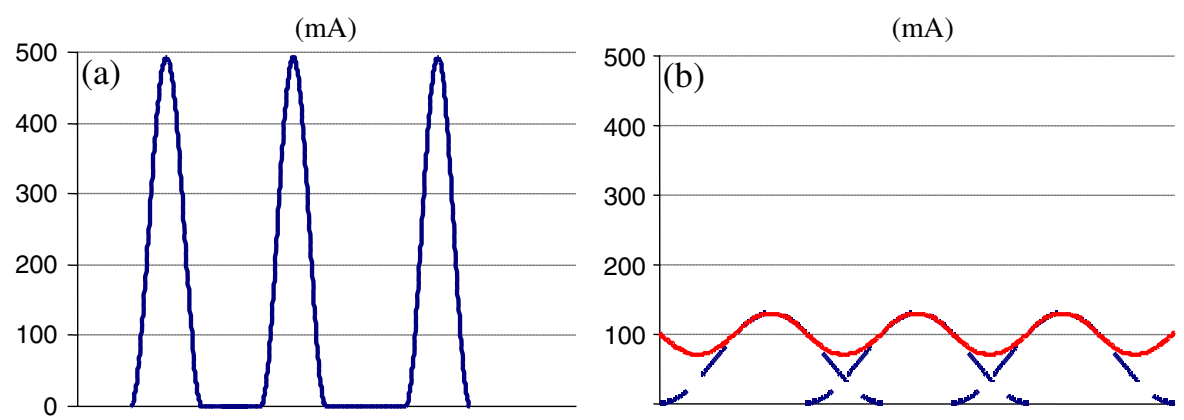

FIG. 8. (Color) (a) The MATHEMATICA simulation of the IPHI beam current versus time, the beam is bunched, the mean current is $100 \mathrm{~mA}$. (b) In the dashed curve, the corresponding image signal parts of the bunched beam, seen at the wall, lead to a superposition, while the mean value (DC component) is still $100 \mathrm{~mA}$. The AC components are reduced, the red trace looks like a sine function, and therefore the current out of the button has very few harmonics.

TABLE II. The wall current amplitude of the first Fourier harmonic (352.2 MHz) simulated for each BPM and for two bunch distributions. The BPM are designed for a $33 \mathrm{~mm}$ chamber radius and the last one for a $75 \mathrm{~mm}$ chamber radius.

\begin{tabular}{|c|c|c|c|c|c|c|}
\hline \multirow[b]{2}{*}{ BPM number } & \multirow[b]{2}{*}{$\sigma_{\text {beam }}$ in $\mathrm{mm}$} & \multirow[b]{2}{*}{$\sigma_{\mathrm{im}}$ in $\mathrm{mm}$} & \multicolumn{2}{|c|}{ Cosine $^{2}$ distribution } & \multicolumn{2}{|c|}{ Gaussian distribution } \\
\hline & & & $A_{1}$ (i wall) in $\mathrm{mA} \sim$ & $\mathrm{v}_{\text {button }}(\mathrm{dBm})$ & $A_{1}$ (i wall) in $\mathrm{mA} \sim$ & $\mathrm{v}_{\text {button }}(\mathrm{dBm})$ \\
\hline $1 \mathrm{st}$ & 6 & 24 & 16.5 & -5 & 17.0 & -5 \\
\hline 2nd & 14 & 27 & 4.5 & -16 & 8.8 & -11 \\
\hline $3 \mathrm{rd}$ & 15 & 28 & 4.9 & -16 & 7.8 & -12 \\
\hline 4th & 17 & 55 & 0.7 & -38 & 0.0 & -103 \\
\hline
\end{tabular}

A more complete calculus of the wall current on the electrode is given by Shafer's paper [9], where a Fourier decomposition of the current beam is required. A weakness of the Shafer formula is that it is only valid in the electrostatic case. A new MATHEMATICA program developed integrates all harmonics of the wall current along the button electrode, based on the formula given by Shafer.

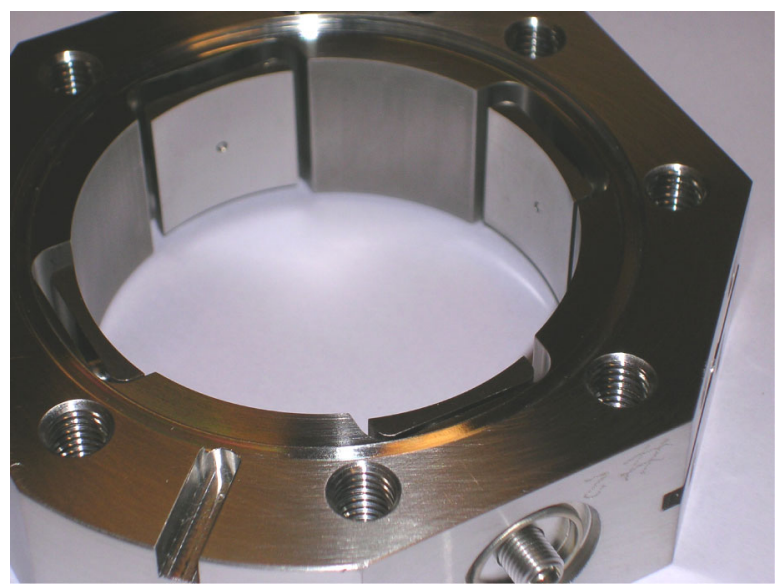

FIG. 9. (Color) An IPHI BPM (core, feedthrough, and assemblies) has been built by the French company PMB. The space between electrode and core is $2 \mathrm{~mm}$ with a tolerance of $\pm 0.05 \mathrm{~mm}$. The four standard Metaceram $50 \Omega$ feedthroughs are terminated by SMA connectors. During the brazing process the four electrodes have been positioned by using a template to align the axial symmetry.
Kowina has developed a numerical code based on finite element methods for the design of BPM and for the simulation of its characteristics [10].

\section{CHARACTERIZATION}

\section{A. Beam position monitor}

Figure 9 shows an IPHI BPM picture; the electrode dimensions are $L=22 \mathrm{~mm}$ and $\operatorname{arc}_{\text {button }}=22 \mathrm{~mm}$, corresponding to the maximum dimensions which can be supported by the $0.92 \mathrm{~mm}$ diameter molybdenum central rod of the Metaceram standard feedthrough. The electrode capacitances at low frequency were measured with a LC

TABLE III. Measured capacitances of the electrodes. The measurement was performed from the SMA connector with a LC meter, $1.4 \mathrm{pF}$ capacitance of the feedthrough itself has been deduced. Only small differences of the capacitance values measured at the same BPM block were observed.

\begin{tabular}{lrrrrc}
\hline \hline $\begin{array}{l}\text { BPM } \\
\text { number }\end{array}$ & \multicolumn{5}{c}{$\begin{array}{c}\text { Capacitance of the } \\
\text { four electrodes in PF }\end{array}$} \\
\hline Prototype & $\mathbf{1 0 . 5}$ & $\mathbf{1 0 . 8}$ & $\mathbf{1 0 . 7}$ & $\mathbf{1 0 . 8}$ & $\begin{array}{c}\text { Difference } \\
\text { in \% }\end{array}$ \\
0\# & $\mathbf{4 . 9}$ & $\mathbf{4 . 9}$ & $\mathbf{4 . 8}$ & $\mathbf{4 . 8}$ & $\mathbf{0 . 3}$ \\
$\# 1$ & $\mathbf{4 . 8}$ & $\mathbf{4 . 8}$ & $\mathbf{4 . 8}$ & $\mathbf{4 . 8}$ & $\mathbf{1 . 6}$ \\
$\# 2$ & $\mathbf{4 . 8}$ & $\mathbf{4 . 8}$ & $\mathbf{4 . 8}$ & $\mathbf{4 . 8}$ & $\mathbf{0 . 5}$ \\
$\# 3$ & $\mathbf{5 . 0}$ & $\mathbf{4 . 9}$ & $\mathbf{4 . 9}$ & $\mathbf{4 . 9}$ & $\mathbf{1 . 6}$ \\
$\# 4$ & $\mathbf{4 . 9}$ & $\mathbf{4 . 9}$ & $\mathbf{4 . 8}$ & $\mathbf{4 . 9}$ & $\mathbf{1 . 6}$ \\
$\# \mathbf{5}$ & 4.7 & 4.6 & 4.9 & 4.9 & 4.8 \\
\hline \hline
\end{tabular}


meter and are listed in Table III. The capacitance of the feedthrough itself has been subtracted. The electrode capacitance deduced from the frequency measurement with the adapted tube is $4.5 \mathrm{pF}$ (Fig. 3).

The weak difference of the measured button capacitances in a BPM block (Table III) was obtained by the technique of the industrial PMB Company. A template is used to guarantee the symmetrical positions of the four electrodes during the brazing procedure.

\section{B. Read-out electronics}

The voltage signals from the button electrodes are processed by the commercial Bergoz log-ratio beam position monitor (LR-BPM) electronics module. The LR-BPM gives the transverse centroid position of the beam from the logarithm of opposite button signals [11]. The signal processing of these modules, operating in track-continuous mode, is compatible with a low duty cycle pulse operation during commissioning of the machine. The duration of the IPHI beam pulse can be as short as $300 \mu \mathrm{sec}$. The fast analogue processing of the LR-BPM modules reports in all conditions the actual beam position, which is more convenient during these initial tests, than using the multiplexed MX Bergoz module [11]. After this period, a compatible MX-BPM could be used in CW mode to reach a higher spatial position resolution. The input bandpass filter frequency is specified for $352.2 \mathrm{MHz}$. The rf input dynamic range is $-70 \mathrm{dBm}$ (or less) to $+5 \mathrm{dBm}$, nevertheless, the strong dependence of the position resolution with the $\mathrm{rf}$ input level will be discussed in III B 1. The LR-BPM provides two continuous analogue voltages, corresponding to $X$ and $Y$, with a bandwidth of $5 \mathrm{MHz}$ in the range $\pm 10 \mathrm{~V}$.

\section{Resolution}

The position resolution is deduced from the noise of the front-end electronics described Fig. 10.

The voltage noise generator $e_{n}{ }^{2}\left(\mathrm{~V}^{2} / \mathrm{Hz}\right)$ is transformed to an equivalent current noise generator $i_{n s}{ }^{2}\left(\mathrm{~A}^{2} / \mathrm{Hz}\right)$,

$$
i_{n s}^{2}=\frac{e_{n}^{2}}{\left|R_{0}+Z_{\text {source }}\right|^{2}} .
$$

For the parallel noise $i_{n}{ }^{2}\left(\mathrm{~A}^{2} / \mathrm{Hz}\right)$, the noise current flowing in the preamplifier is

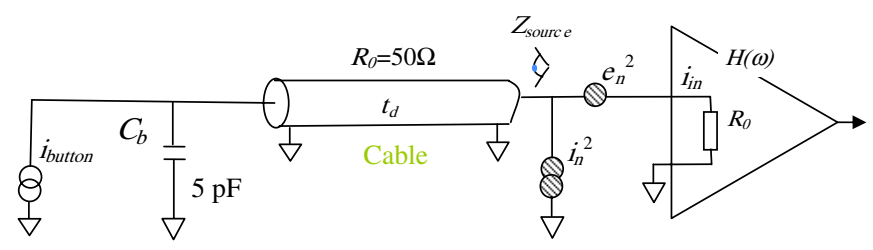

FIG. 10. (Color) Equivalent front-end electronics circuit for noise analysis. $i_{n}$ and $e_{n}$ are parallel and series sources of the noise. $H(\omega)$ is the transfer function of the amplifier.

$$
i_{n p}^{2}=i_{n}^{2}\left|\frac{Z_{\text {source }}}{R_{0}+Z_{\text {source }}}\right|^{2}
$$

The total rms noise at the output of the amplifier is given by

$$
v_{\text {out }}^{2}=\left(i_{n s}{ }^{2}+i_{n p}{ }^{2}\right) R_{0}{ }^{2}|H(j \omega)|^{2} .
$$

If the transmission line (cable) is ideal, the impedance source at the end is given by

$$
Z_{\text {source }}=\frac{\frac{1}{j C_{d} \omega}+j R_{0} \operatorname{tg}\left(\omega t_{d}\right)}{1+\frac{t g\left(\omega t_{d}\right)}{R_{0} C_{d} \omega}} .
$$

References [12,13] presented in more detail the calculated noise depending on the transmission line terminated by a capacitive detector. Figure 11 shows the dependence of $Z_{\text {source }}$ with the transmission line length. At a given frequency, the source impedance can take any values for a short cable; for a long one the impedance observed goes to $50 \Omega$.

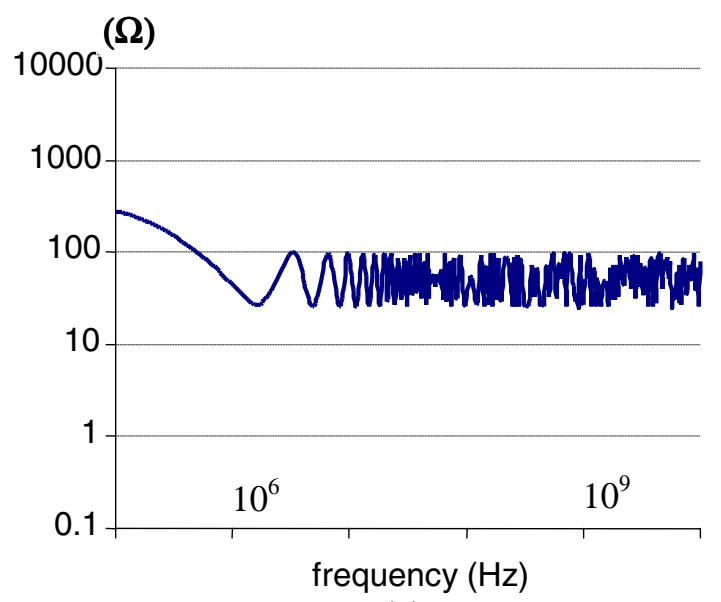

(a)

$(\Omega)$

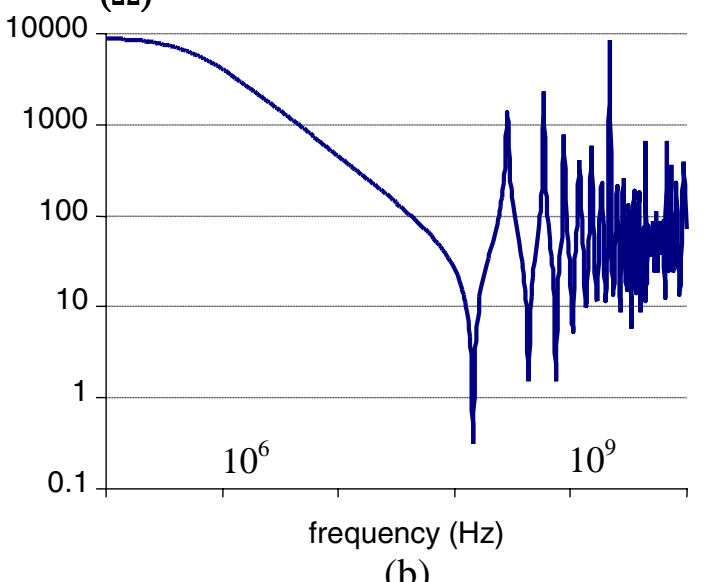

(b)

FIG. 11. (Color) SPICE AC analysis of the source impedance $Z_{\text {source }}$ versus frequency for (a) $30 \mathrm{~m}$ and (b) $30 \mathrm{~cm}$ lengths of the RG-58 cable. For a capacitive BPM pickup, it is better to implement long transmission lines to match the impedances. 
The cable is also used as a parasitic filter, by choosing its equivalent bandwidth close (a little larger) to the processing frequency of the readout electronics.

The normalized positions $x$ and $y$ are obtained by a centroid of the buttons voltages $v_{i}$. For a symmetric pickup with circular cross section, rotated by $45^{\circ}$,

$$
\begin{aligned}
& x=\frac{\left(v_{A}+v_{D}\right)-\left(v_{B}+v_{C}\right)}{\sum_{i=A}^{D} v_{i}}, \\
& y=\frac{\left(v_{A}+v_{B}\right)-\left(v_{C}+v_{D}\right)}{\sum_{i=A}^{D} v_{i}} .
\end{aligned}
$$

The spatial resolution is given by the variances $V(x)$ and $V(y)$ which are the quadratic derivation of the centroid:

$$
V(x)=\frac{1+x^{2}}{\left(\sum_{i=A}^{D} v_{i}\right)^{2}} \sigma v_{i}^{2},
$$

same for $y$. In this formula the noise $\sigma v_{i}$ in each electrode is supposed to be the same. The spatial resolution depends on the position itself, the optimum is at the center of the BPM. The position $X$ (in $\mathrm{mm}$ ) is obtained by multiplying the normalized position $x$ by a coefficient $K_{x}$ which is directly proportional to the size of the chamber. Thus, the spatial resolution (in $\mathrm{mm}$ ) is also directly proportional to the radius pickup. The last and main consideration is that the spatial resolution is inversely proportional to the sum of the four electrode voltages. The resolution of the BPM increases, as the beam intensity, i.e., signal levels of the electrodes are shown in Fig. 12.

The LR-BPM bandwidth is about $10 \mathrm{MHz}$; it is recommended for improving spatial resolution to add an antialiasing filter before the sampling of the LR-BPM output

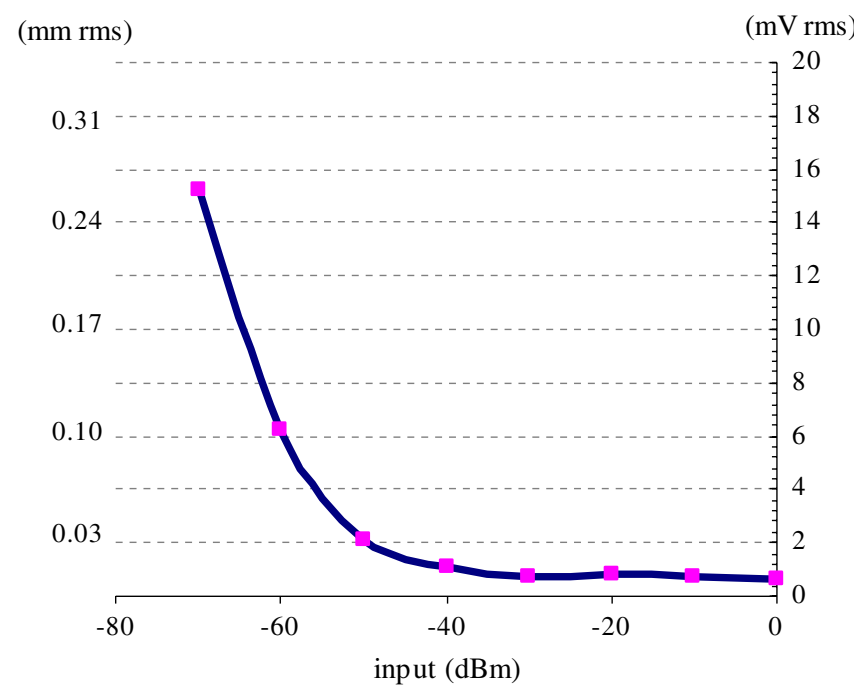

FIG. 12. (Color) The spatial resolution depends on the beam intensity. The right scale is the noise measured after the sampling of the output of the LR Bergoz module with the $6052^{\mathrm{E}}$ PXI card. The left scale is the equivalent spatial noise for a chamber of $33 \mathrm{~mm}$ radius. signal. The $6052^{\mathrm{E}}$ input amplifier has a bandwidth of $480 \mathrm{kHz}$ and is used as a filter. The numerical processing is based on taking the mean value of the samples, thus the sigma of the position is reduced in root square of the samples number.

The measurement of the long term repeatability gives $11 \mu \mathrm{m} \mathrm{rms}$ in CW mode and $13 \mu \mathrm{m}$ rms in $300 \mu$ s pulsed mode. For this stability measurement, the temperature is also measured and only data taken at the same temperature have been considered.

\section{Temperature}

The AD8306 logarithmic amplifier chip used in the LRBPM has a temperature drift of $-0.1 \%$ per degree. The drift given for the LR-BPM module is $41 \mu \mathrm{m} / \mathrm{K}$ and depends of the relative variations between two channels. The worst case measured among six modules is $-40 \mu \mathrm{m} / \mathrm{K}$. This is not acceptable in our application; moreover, the IPHI experimental hall is not temperature controlled, therefore a temperature regulation of the LR Bergoz module was implemented (Fig. 13). A simple fan was placed on the Bergoz crate, closer to the input amplifier. After testing various regulation systems, an on-off regulation has been adopted because a high gain is needed to rapidly extract the hot air generated by the Bergoz module.

This regulation was tested with a room temperature ranging from $13^{\circ} \mathrm{C}$ to $29^{\circ} \mathrm{C}$, the LR-BPM modules are regulated at $0.7^{\circ} \mathrm{C}$. For this range, the measured position drift is less than $20 \mu \mathrm{m}$. The performance of the BPM electronics is summarized in Table IV.

\section{Sensitivity}

In the electrostatic case there is a linear dependence between $Z_{c}$ (Sec. II A) and the electrode voltage. Therefore, any difference in dispersion between the four cables leads to an offset of the reported beam position. This is even more relevant, as the $20 \mathrm{~m}$ long cables are not terminated at both ends in their characteristic impedance. At the upstream end, the cable is terminated by the electrode which has a capacitance value of $4.8 \mathrm{pF}$. At the downstream end, the logarithmic amplifier (LR-BPM) has an input impedance complex $R+1 / j \omega C=$ $28-j 26 \Omega$ at $352 \mathrm{MHz}$ instead of the characteristic impedance $50 \Omega$. This causes standing waves in the cable, which increases the offset effect due to the difference in dispersion of $Z_{c}$ between the transmission lines. A dispersion of $2 \%$ in $Z_{c}$ between two cables is a typical value corresponding to an offset shift of $800 \mu \mathrm{m}$.

The dispersion of $Z_{c}$ was measured for five types of cables. For the characterization of the BPM offset, the Hytem 402 semirigid cable, which has the lowest dispersion $( \pm 0.2 \%)$, is used. The experimental test shows the dependence of offset error on the cable length because of the standing waves ratio. A very long cable attenuates this 


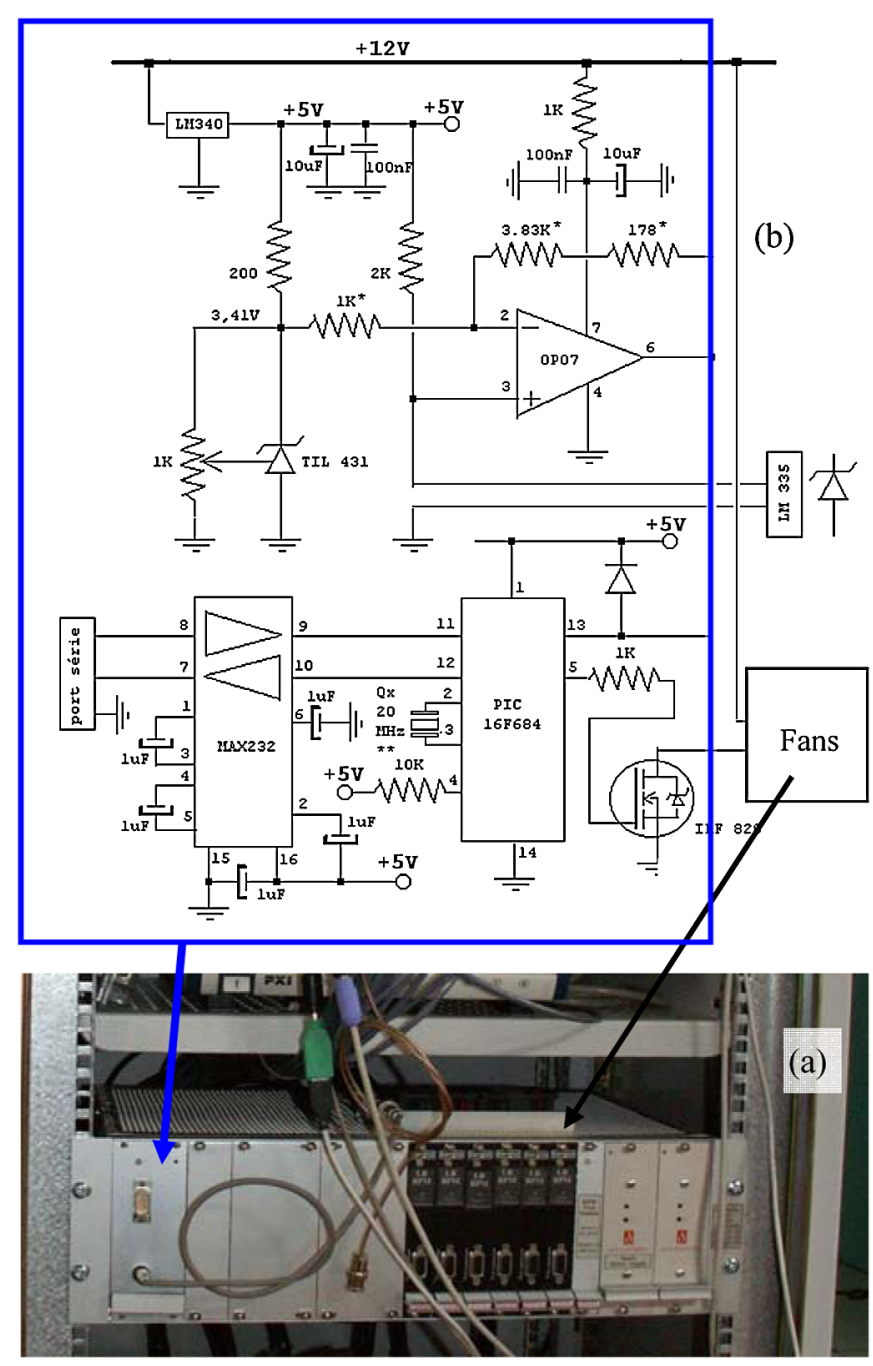

FIG. 13. (Color) (a) The Bergoz crate with the six LR modules with black front panels. (b) The schematic of the temperature regulation that is implemented in the module on the left position of the crate. The LM335 temperature sensor is located in the LR module, close to the amplifier input. A film covers the top of the Bergoz crate isolating it thermically. The two fans extract the hot air produced by the electronics.

effect. The offset effect can also be minimized by matching the electric length of the four cables. As the electrical length depends on cable bends, temperature, etc., an in situ calibration is required.

The determination of the position sensitivity is essential, as it determines some requirements of the subsystems, e.g., electric length match of the cables, accuracy of the readout electronics, tolerances of the BPM pickup mechanics, etc., to ensure the expected performance of the complete BPM system.

The intrinsic chamber sensitivity is the variation in percent or in $\mathrm{dB}$ between two signals of opposite electrodes for $1 \mathrm{~mm}$ of beam transverse displacement. For low beta beam, the sensitivity could not be measured with a wire displacement in the laboratory because $\beta=1$. The sensitivity is determined by a field simulation or by following the Shafer formula [9] based on the Cupérus paper [14]. The formula was computed with MATHEMATICA. An approximation for small displacement is also given in [9] and can be calculated by hand. For IPHI BPM the sensitivity measurement on the bench test $(0.98 \mathrm{~dB} / \mathrm{mm})$ is in good agreement with the calculation $(1.03 \mathrm{~dB} / \mathrm{mm})$. This measurement obtained with a scope can be improved by using a power meter. For $\beta=0.04$ the calculation gives $1.93 \mathrm{~dB} / \mathrm{mm}$ corresponding to a signal variation of $1.25 / \mathrm{mm}$. On the other side this also means a gain difference (error) in the signal processing of $2.5 \%$ corresponds to a position error of $100 \mu \mathrm{m}$. The characterization and calibration with a test stand introduces several error sources (see Sec. IV and [15]). Each error should be smaller than the total accuracy requirement. In IPHI we aim an accuracy of about $\pm 0.25 \%$ for each error source.

The test stand measurements are valid for $\beta=1$. For low velocity beam (low $\beta$ ), the positions measured at frequency $f$ of a chamber of radius $a$ are multiplied by $(1+G)$, where [9]

$$
G=0.139\left(\frac{2 \pi f a}{\beta \gamma c}\right)^{2}-0.0145\left(\frac{2 \pi f a}{\beta \gamma c}\right)^{3} .
$$

\section{TEST STAND}

The test stand is used to characterize the BPM and calibrate the complete electronics chain. The stand consists of a coaxial line of $189 \Omega$ characteristic impedance. An Agilent $8648 \mathrm{C}$ generator drives a rod (center conductor) of $2.05 \mathrm{~mm}$ diameter at a frequency of $352.2 \mathrm{MHz}$. The $66 \mathrm{~mm}$ diameter pipe is fixed on an $X-Y$ positioning table driven by stepper motors, as we can see on Fig. 14. The rod is fixed and tensioned with nuts through the drilled printed circuits. The printed circuit boards match the impedances

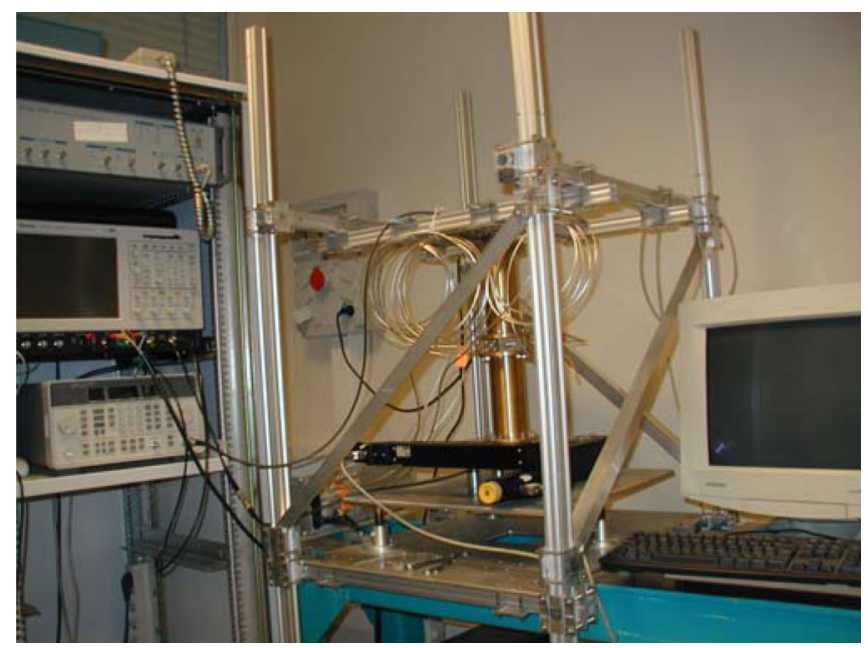

FIG. 14. (Color) Photo of the test stand, dedicated for mapping, characterization, and calibration. 


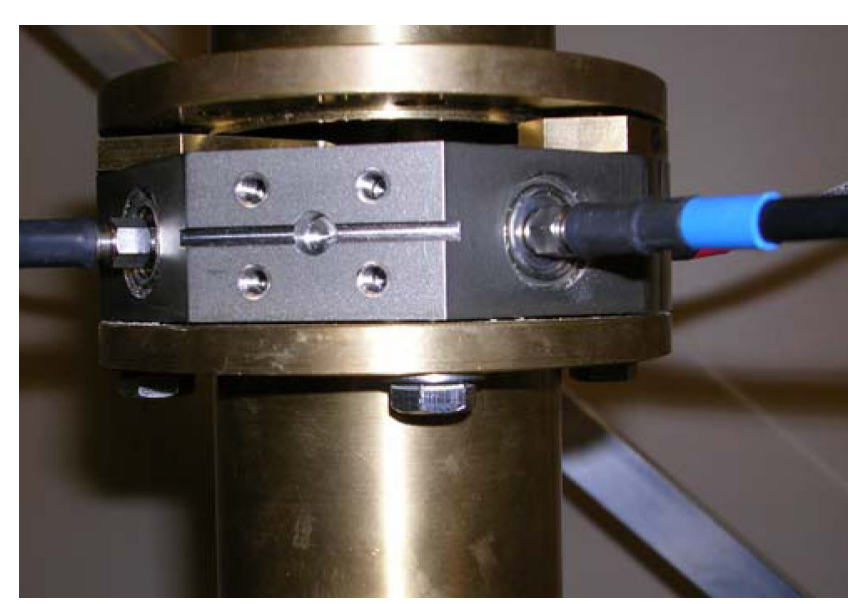

FIG. 15. (Color) Photo of the template positioned with pawns above the BPM block. The rod is positioned at the center of the BPM in $X$ and $Y$ by electric contacts with the template. Afterwards, the template is removed and the upper flange is delicately screwed on the BPM. Two half crown pieces are inserted under the rf joint to maintain an equal height.

with resistor networks at both ends. The signal is returned to the flanges via two HF joints which slide without constraint on the other side of the boards. The joints also serve to protect the interior of the tube from $\mathrm{rf}$ interference. The hysteresis error of the mechanical system is $15 \mu \mathrm{m}$.

The rod is easily removed by unscrewing nuts on each side causing the rod to drop through the hole of the printed circuit.

The determination of the absolute rod position is performed by an electrical contact (accuracy $\pm 2.5 \mu \mathrm{m}$ ) with a template, shown in Fig. 15, inserted onto the BPM and centered with pawns. The mechanical dimensions of the template are controlled to $\pm 5 \mu \mathrm{m}$ by the measuring system Mitutoyo EURO-M574. After alignment of the rod to the center of the BPM under test, the template is removed and the upper flange is carefully screwed on the BPM.

The positioning error of the two flanges results in a measurement error of $\pm \sqrt{2} \times 25 \mu \mathrm{m}$, this error is divided statistically by the square root of the number of performed calibration procedures. A mechanical deformation was measured after the BPM screwing on the flanges corresponding to a coaxiality error of $35 \mu \mathrm{m}$ between the rod and the BPM.

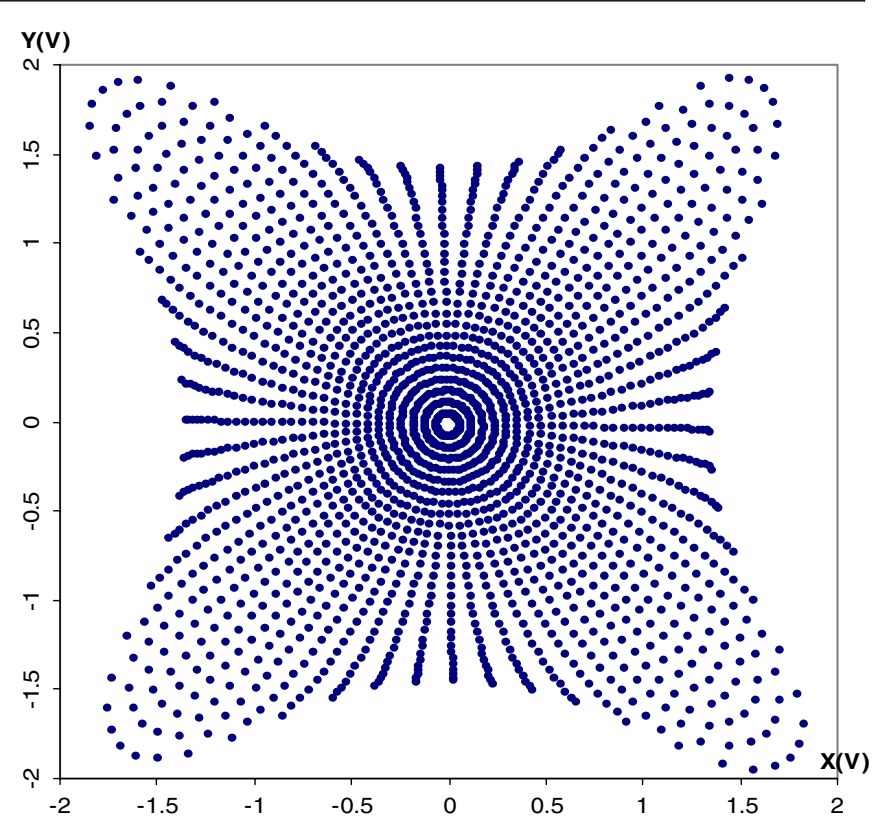

FIG. 16. (Color) Mapping of the BPM position characteristics moving the stimulus (rod center conductor, $i_{\text {rod }}=0.3 \mathrm{~mA}$ ) along polar coordinates. The radius was incremented by $1 \mathrm{~mm}$. $X$ and $Y$ are directly the measured positions by the log-ratio Bergoz module.

The error sources of the mechanical system including the calibration are summarized in Table VI in Sec. V.

The acquisition system is based on the peripheral component interconnect extensions for instrumentation (PXI) hardware standard. The stepper motors are driven by a National Instruments stepper driver unit type NI MID7604, which is controlled by a PXI stepper motion controller (type NI PXI-7334) from the same company. A LABVIEW program developed in-house controls the hardware and perform the data acquisition.

To characterize spatial linearity, the rod is positioned at the mechanical center and, for different radial positions the corresponding electrical positions are measured directly with the LR Bergoz module. Figure 16 shows the mapping corresponding to this measurement realized with the temperature regulation described previously.

Figure 17 shows the dynamic range of the LR-BPM module with respect to beam displacement and intensity

TABLE IV. Performance of the BPM readout electronics.

\begin{tabular}{lcccc}
\hline \hline Electronics & \multicolumn{2}{c}{ Error in $\mu \mathrm{m}$ for different input power } & Remarks \\
\hline & $>-33 \mathrm{dBm}$ & $-60 \mathrm{dBm}$ & $-70 \mathrm{dBm}$ & \\
\hline Noise & $15.8 \mathrm{rms}$ & $124 \mathrm{rms}$ & $1674 \mathrm{rms}$ & Centroid is intensity dependant \\
$\sigma$ of the mean value & $3.2 \mathrm{rms}$ & $25.3 \mathrm{rms}$ & $342 \mathrm{rms}$ & 12 points in $300 \mu$ s pulsed mode \\
Stability & $12.6 \mathrm{rms}$ & Not tested & Not tested & Long term repeatability \\
Temperature & $15.5 \mathrm{max}$ & Not tested & Not tested & Temperature regulated \\
\hline \hline
\end{tabular}




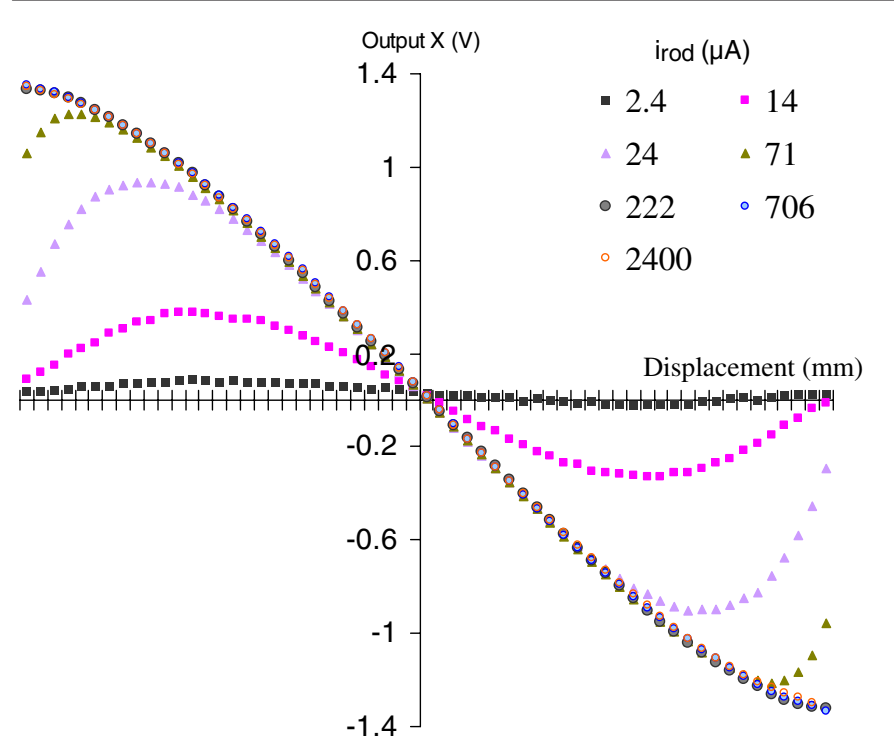

FIG. 17. (Color) $X$ response of the BPM reading with the LRBPM versus the rod displacement. The values of the current take into account the correction with $\beta$ (Sec. II A). For a current above $10 \mu \mathrm{A} \mathrm{AC}$, the nonlinearities are acceptable and the beam can be centered by iteration with a precision of $100 \mu \mathrm{m}$.

variation; at lower beam currents $(<100 \mu \mathrm{A})$ we observe substantial nonlinearities (drop of gain).

The total gain of BPM with its electronics is $61.3 \mathrm{mV} / \mathrm{mm}$ until an input power of $-60 \mathrm{dBm}$. This gain becomes $30.7 \mathrm{mV} / \mathrm{mm}$ for an input power of $-70 \mathrm{dBm}$ and $8.4 \mathrm{mV} / \mathrm{mm}$ for $-80 \mathrm{dBm}(-80 \mathrm{dBm}$ corresponds to a current seen by the electrode of $10 \mu \mathrm{A}$ $\mathrm{AC})$. When an off-center beam is reported, the steerer moves it back close to the center. The beam converges to the center by iterations between the position measurements and the current applied to the steerer and it is not necessary to have a sensitivity calibration with the intensity. IPHI will be used for high intensity; nevertheless the convergence is possible up to $10 \mu \mathrm{A}$ with an acceptable precision. The alignment accuracy of the accelerator component is a necessary condition and the calibration of the offset is required.

\section{OFFSET CALIBRATION}

The calibration is performed in two steps: first, for the determination of the intrinsic offset of the BPM block itself. This offset is due to unsymmetrical positions of the electrodes and the relative difference of the buttons capacitances. The intrinsic offset $\left(\Delta x_{0}, \Delta y_{0}\right)$ is a constant. The second step consists, in laboratory and in situ, of the offset measurement of the LR electronics module and associated cables $\left(\Delta x_{c}, \Delta y_{c}\right)$. This offset is due to the difference in impedance and gain between channels.

In running, the total offset $\left(\Delta x_{0}+\Delta x_{c}, \Delta y_{0}+\Delta y_{c}\right)$ is subtracted from the measured beam position as explained in Fig. 19.

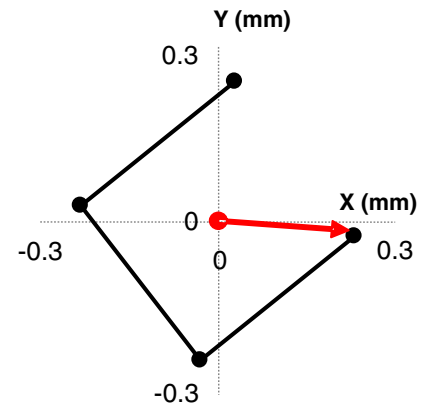

FIG. 18. (Color) Each dot is one measurement. The intrinsic offset $\left(\Delta x_{0}, \Delta y_{0}\right)$ is represented by the vector. The circular rotation is performed by switching the cable on the upstream BPM side to the same readout channel. For the position measurement the Bergoz precision MX module is used.

\section{A. Intrinsic BPM offset}

The surface reference of the BPM is not the same as the template. The mechanical tolerance between the two references adds an error in the range of $\pm 25 \mu \mathrm{m}$. Asymmetries between the electrodes introduce an intrinsic offset, that is measured using the Bergoz MX precision BPM readout module [11]. As this is a single channel receiver, offset effects due to gain mismatches, etc. are excluded, the signal levels of the four BPM electrodes are detected sequentially by permutation (switching). Any differences between the four measurements are due to the block itself and to the positional inaccuracy of the rod. Figure 18 shows an intrinsic offset measurement $\left(\Delta x_{0}, \Delta y_{0}\right)$.

As discussed, changes in the cable runs (bending radius) also have an impact on the offset. Another more difficult problem concerns the repeatability of the measurement after a complete dismount and remount of the BPM block. We obtained accuracy statistics by 16 measurements with a complete dismount for each BPM. The repeatability error and the offsets carried and listed Table V. The offset is determined by the median value of the 16 measurements. The error due to the repeatability is about $35 \mu \mathrm{m} \mathrm{rms}$.

Except for the BPM prototype that has a different mechanical conception, we notice a correlation between the

TABLE V. Intrinsic offsets for each BPM. These values are the result of 16 measurements with a complete dismount and remount of the BPM block. The typical sigma value is $35 \mu \mathrm{m} \mathrm{rms}$.

\begin{tabular}{lrrcc}
\hline \hline BPM & $\begin{array}{c}\Delta x_{0} \\
\text { number }\end{array}$ & $\begin{array}{c}\Delta y_{0} \\
\text { in } \mathrm{mm}\end{array}$ & $\begin{array}{c}\sigma_{\Delta x 0} \\
\text { in } \mathrm{mm}\end{array}$ & $\begin{array}{c}\sigma \Delta y 0 \\
\text { in } \mu \mathrm{m}\end{array}$ \\
\hline Prototype & $\mathbf{0 . 2 5 9}$ & $\mathbf{0 . 0 7 2}$ & $\mathbf{3 6}$ & 46 \\
$\# 0$ & $\mathbf{0 . 0 0 8}$ & $\mathbf{- 0 . 0 1 0}$ & $\mathbf{3 5}$ & 48 \\
$\# 1$ & $\mathbf{- 0 . 0 0 8}$ & $\mathbf{0 . 0 1 6}$ & $\mathbf{1 4}$ & 39 \\
$\# 2$ & $\mathbf{- 0 . 0 0 4}$ & $\mathbf{- 0 . 0 1 6}$ & $\mathbf{4 3}$ & 19 \\
$\# 3$ & $\mathbf{- 0 . 0 5 4}$ & $\mathbf{- 0 . 0 4 4}$ & $\mathbf{2 9}$ & 34 \\
$\# 4$ & $\mathbf{- 0 . 0 1 1}$ & $\mathbf{- 0 . 0 3 0}$ & $\mathbf{3 6}$ & 23 \\
$\# \mathbf{5}$ & $\mathbf{- 0 . 0 0 0}$ & $\mathbf{0 . 1 5 9}$ & 40 & 41 \\
\hline \hline
\end{tabular}


offset and the dispersion of the four capacitances given in Table III. The BPM \#5 has the largest offset and the largest relative capacitance difference.

\section{B. Cable and electronics offset}

A coaxial cable type LMR 240 is installed to connect the BPM electrodes to the Bergoz readout electronics. The offset due to the LR-BPM electronics and its associated cables is determined with the precedent principle, the permutation of the input cables on the BPM side. "Rotating" the cable connections of the four inputs is "equivalent" to a mechanical rotation of the BPM block. The results of $\left(\Delta x_{c}, \Delta y_{c}\right)$ are given in Fig. 19. The high dispersion values of the electronics offset are caused by the logarithmic amplifier and the dispersion of the input impedance of the LR-BPM. The total offset is the sum of $\left(\Delta x_{0}, \Delta y_{0}\right)$ and $\left(\Delta x_{c}, \Delta y_{c}\right)$ as shown Fig. 19.

The cables and the electronic offset $\left(\Delta x_{c}, \Delta y_{c}\right)$, as the input electronics is a logarithmic amplifier, are also strongly dependent on the input power and also on the beam intensity. For each channel, ten values of $\left(\Delta x_{c}, \Delta y_{c}\right)$ are stored as a look-up table for the entire dynamic range. The offset applied is given by the $\Sigma$ signal measured from the LR-BPM [11]. The analogue signal $\Sigma$ is a continuous value proportional to the sum of the signals from the four buttons which are proportional to the beam intensity.

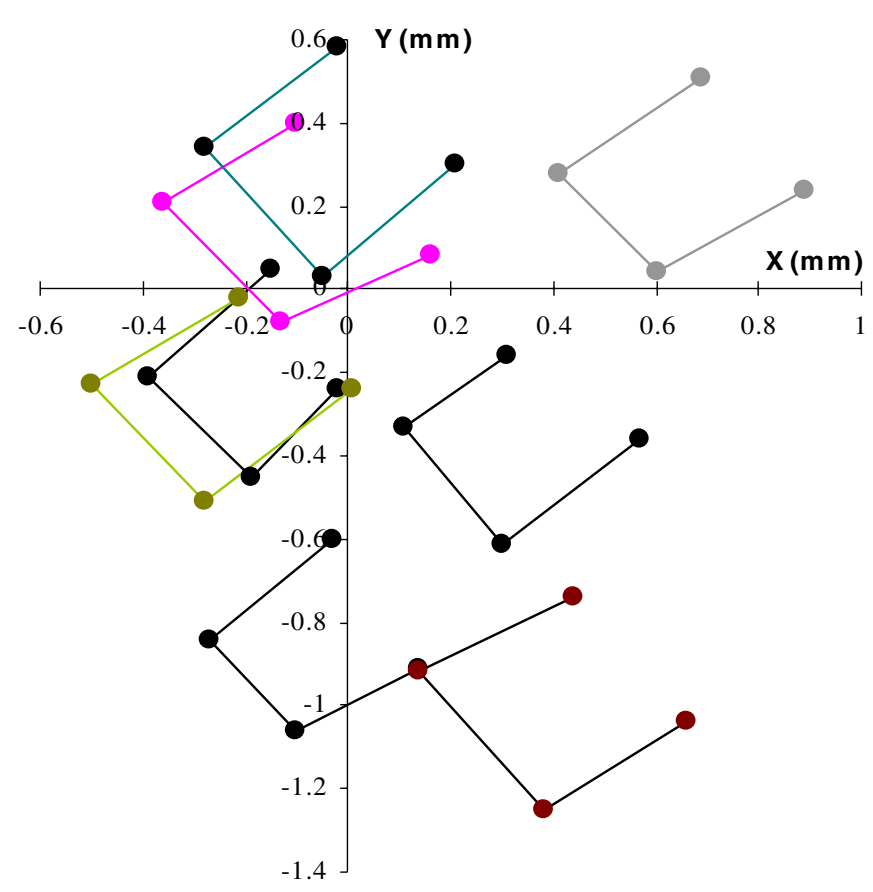

FIG. 19. (Color) Total offset measured with eight different LRBPM electronics channels. Each dot corresponds to one measurement. The cable ends at the side of the BPM are permuted 4 times. The red vector corresponds to the intrinsic offset and the blue vector corresponds to the offset $\left(\Delta x_{c}, \Delta y_{c}\right)$ associated to the cables and the electronics.

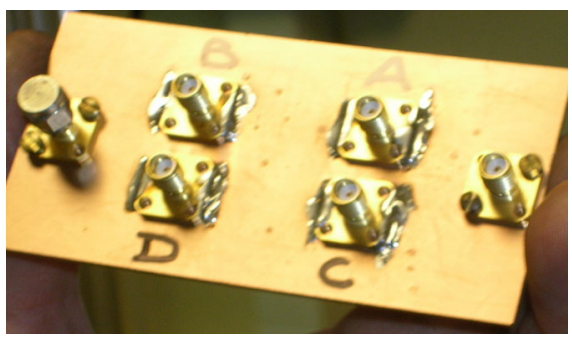

FIG. 20. (Color) The fake block simulates a BPM block response to a centered beam. The fake block is driven by a rf generator to allow cable and electronics calibration after installation in site without the beam.

Between two recorded points a linear interpolation is applied with a maximum error of $32 \mu \mathrm{m}$.

$\left(\Delta x_{c}, \Delta y_{c}\right)$ depends on the cable bends and therefore on their installation on the accelerator site. Cables and electronics calibration was performed before and after the installation on site without the beam. This calibration is based on the $\left(\Delta x_{c}, \Delta y_{c}\right)$ measurement by replacing the BPM block by a "fake block." The fake block is a printed circuit board (Fig. 20) with four matched capacitors of value $C_{b}$. The signal from a generator $352 \mathrm{MHz}$ drives a strip line which is coupled to the four capacitances. This fake block simulates the response of a BPM to a centered beam.

Six strands of four cables LM 240 are installed on site. Their length range is from 15 to 20 meters.

\section{Embedding}

The difference of the response of the fake block and the real BPM block mounted on the test stand was characterized in the laboratory. A typical measurement result is plotted in Fig. 21.

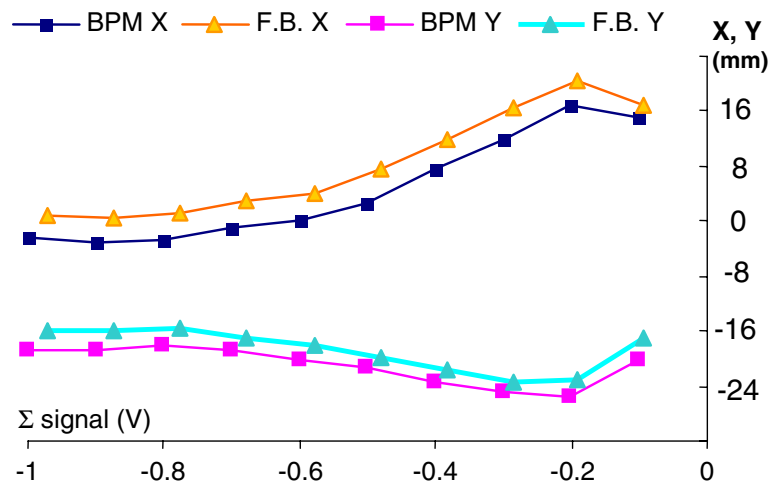

FIG. 21. (Color) Comparison of $X$ and $Y$ position measurements for the fake block (F.B.) and the BPM block mounted on the test stand. The responses are given versus the sum from the four electrodes. We observe an intensity dependence for the $X$ and $Y$ displacement values due to the limitations of the logarithmic amplifier in the readout electronics. 


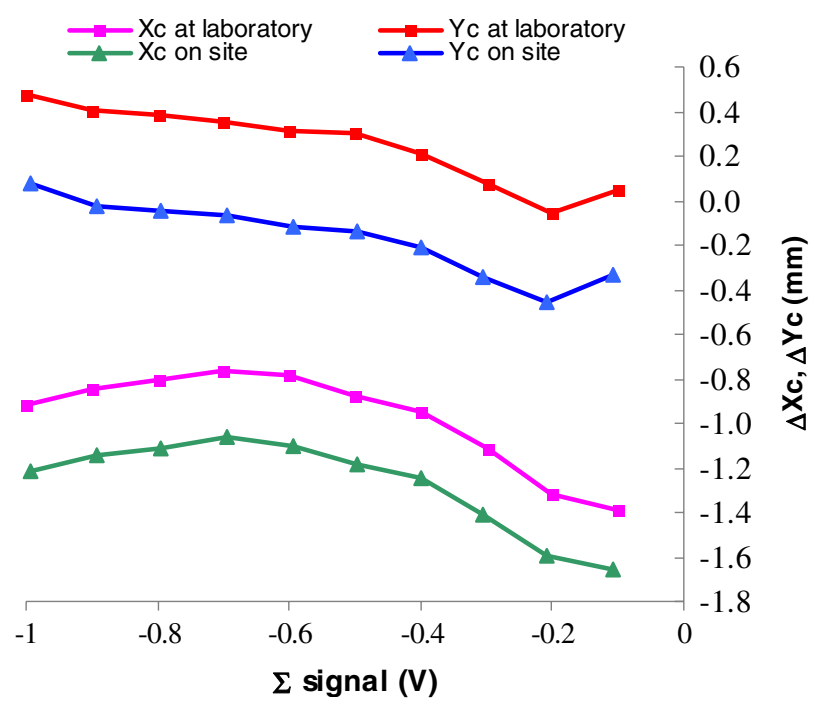

FIG. 22. (Color) Offsets $\left(\Delta x_{c}, \Delta y_{c}\right)$ versus intensity. We compare the measurements performed at the test stand (in laboratory) and after final installation (on site). The cable length used for the test stand was 19.10 meters. The major difference between the measurements is due to the different bendings of the coaxial cables running between the BPM block and the readout electronics.

The difference between the two traces is a constant versus the intensity and is called the residual offset $\left(\Delta x_{R}, \Delta y_{R}\right)$. This residual offset, as a result of the difference between the BPM block and the fake block, is characterized in the laboratory and is subtracted from the total offset. Four identical capacitors of the value $C_{b}$ are imple- mented on the fake block but any other values of the four impedances could be implemented and would result in an increase of the constant $\left(\Delta x_{R}, \Delta y_{R}\right)$, which could also be characterized.

The offset $\left(\Delta x_{c}, \Delta y_{c}\right)$ was measured with the fake block before and after the installation. Figure 22 shows the $\left(\Delta x_{c}, \Delta y_{c}\right)$ measurements in the laboratory and after the installation on site. The difference is due to the cable bends.

To summarize, the beam position is given by the following formula:

$$
\begin{aligned}
\text { beam position }= & \text { read position }-\left(\Delta x_{0}, \Delta y_{0}\right) \\
& -\left(\Delta x_{c}, \Delta y_{c}\right)-\left(\Delta x_{R}, \Delta y_{R}\right) .
\end{aligned}
$$

The cables and electronics calibration could also be performed with the bunch based center (BBC) card from Bergoz [11]. The BBC short circuits the four cable inputs by coupling to have four identical signals for any transverse position of the beam. The $\mathrm{BBC}$ needs a beam to perform the calibration. The offset of the BBC was tested on the test stand, the residual offset ranging from 200 to $800 \mu \mathrm{m}$ according to the cables and electronics channels. This device was not used because it has to be inserted permanently in series between the BPM block and the four cables, and would add an insertion loss of $10 \mathrm{~dB}$.

Table VI summarizes the results obtained from the positioning system and the calibration.

In order to align the BPM with the beam, a fiducial, fixed onto the BPM, is used. The primary error is due to the BPM block, to be aligned with the other beam line components

TABLE VI. Test stand and calibration error summary.

\begin{tabular}{lcl}
\hline \hline Calibration & Error max in $\mu \mathrm{m}$ & \multicolumn{1}{c}{ Remarks } \\
\hline Centering pin/BPM center & 25 & Tolerance error \\
Flanges alignments & 35.4 & Flanges in contact with the BPM \\
Template dimensions & 5 & The template has been controlled \\
$\Phi$ rod error & 10 & Controlled \\
Electric contact rod/template & 2.5 & $=$ one half step motion table \\
Rod coaxiality & 35 & Estimated by the reproducibility \\
Intrinsic BPM offset & 8.8 & $=35 / \sqrt{16}$ \\
$\left(\Delta x_{c}, \Delta y_{c}\right)$ correction & 8 & Reproducibility (bend cables problem) \\
Correction versus intensity & 32 & Interpolation between two calibration points \\
Hysteresis & 15 & Table displacement \\
\hline \hline
\end{tabular}

TABLE VII. The position error of the BPM at the accelerator.

\begin{tabular}{lcl}
\hline \hline Mechanics on accelerator & Error max in $\mu \mathrm{m}$ & \multicolumn{1}{c}{ Remarks } \\
\hline Alignment precision & 100 & The sight support is positioned on the BPM block \\
Sight support dimension & 8 & The support is 400 mm length \\
Sight support verticality & 40 & Flanges in contact with the BPM block \\
Flange alignments & 35.4 & Brass joint flanges \\
Vacuum joints positioning & 63.6 & \\
\hline \hline
\end{tabular}


of the accelerator (Table VII). The second error term is due to the joints positioning on the knives (Table VII). Figure 9 shows the vacuum knife close to the electrodes. The measured error on the test stand is $63.6 \mu \mathrm{m}$ for a joint position asymmetry of $0.1 \mathrm{~mm}$.

It is recommended to design and characterize the complete BPM pickup, including all joints, flanges, etc. The drawback is the need of a vertical wire in the beam pipe, and to center it with enough precision and verify its coaxiality [15]. Another important problem concerns the bending of the cables, they should not be touched and their manipulation must be done via switches. The offset introduced by the switches must be taken into account. It is also important to verify offset measurement by repeating several complete mount/demount procedures of the BPM hardware.

Lambertson suggested a calibration method without wire based on an electric measurement of the difference of the gains between the channels [16]. The results of this method compared with the wire method are shown in [17].

\section{CONCLUSION}

The new designed and calibrated BPM pickups and their electronics are installed on the IPHI accelerator, waiting for the completion of the RFQ. All precautions were taken to reduce parasitic effects from the rf power through grounding elements to the accelerator support structures. A dedicated BPM test stand was developed, allowing a manual characterization of the BPM with very high precision. The expected precision of the alignment of the BPM block on the accelerator is less than $100 \mu \mathrm{m}$. Assuming that errors are independent of each other, they can be added quadratically. The beam position can be measured with an absolute accuracy of $180 \mu \mathrm{m}$. With this first experience, the main accuracy improvement for the Spiral2 project will be the manufacturing of complete BPMs, including their mounted flanges. The test stand described will be automated to characterize a large number of Spiral2 BPMs. The new test setup is under development and uses remote controlled switches for all signal multiplexing. The main difficulty will be the wire alignment at the mechanical center and to verify its symmetry.

\section{ACKNOWLEDGMENTS}

The author wishes to acknowledge J. N. Maymon (now retired) for his efforts to develop the test stand.

[1] P. Strehl, CERN Report No. 87-10, 1986, p. 99.

[2] P. Ausset, J. N. Maymon, N. Rouvière, M. Solal, and L. Vatrinet, in Proceedings of DIPAC 2005 (CERN, Lyon, France, 2005), РОT013, p. 172.

[3] J.C. Denard, Rubrique: Electronique Mesures Report No. Super-ACO /83-69, 1983.

[4] S. R. Smith, Beam Position Monitor Engineering (AIP, New York, 1997), p. 50.

[5] Tektronix, TDR Impedance Measurements: A Foundation for Signal Integrity, http://www.tek.com/applications/ design_analysis/tdr.html.

[6] L. Cassinari, J.-C. Denard, C. Herbeaux, E. Cenni, M. Canetti, F. Gangini, and J.-L. Billaud, in Proceedings of the 11th European Particle Accelerator Conference, Genoa, 2008 (EPS-AG, Genoa, Italy, 2008), MOPD019.

[7] F. Loyer, TATIHOU-98-talk2-PC, internal GANIL presentation, 14076 Caen.

[8] J. Bosser, CERN Report No. CERN-PE-ED 001-92, 1994, p. 106.

[9] Robert E. Shafer, AIP Conf. Proc. 319, 303 (1994).

[10] P. Kowina, P. Forck, W. Kaufmann, T. Weiland, and F. Wolfheimer, in Proceedings of DIPAC09 (CERN, Basel, Switzerland, 2009), MOOC03.

[11] Bergoz Instrumentation, http://www.bergoz.com.

[12] R. L. Chase, C. de La Taille, S. Rescia, and N. Seguin, Nucl. Instrum. Methods Phys. Res., Sect. A 330, 228 (1993).

[13] M. Akopyan, M. Chen, R. Krasnokutsky, R. Shuvalov, and V. Sushkov, Nucl. Instrum. Methods Phys. Res., Sect. A 330, 465 (1993).

[14] J. H. Cupérus, Nucl. Instrum. Methods Phys. Res., Sect. A 145, 219 (1977).

[15] R. B. Shurter, J. D Gilpatrick, J. Ledford, J. O'Hara, and J. Power, CP451, Beam Instrumentation Workshop 1998 (AIP, New York, 1998).

[16] G. R. Lambertson, Lawrence Berkeley Laboratory LSAP Note-5, 1987.

[17] Y. Chung and G. Decker, Offset Calibration of the Beam Position Monitor Using External Means (AIP, New York, 1992), p. 217. 